\title{
ENTRE RUPTURES ÉDUCATIVES ET CLOISONNEMENTS PÉDAGOGIQUES
}

Repenser la lutte contre le décrochage scolaire en lycée professionnel

\author{
Eric Flavier, Joël Gaillard, Sandoss Ben Abid-Zarrouk
}

Association pour la Recherche en Éducation | « Spirale - Revue de recherches en éducation »

$2017 / 1 \mathrm{~N}^{\circ} 59$ | pages 27 à 39

ISSN 0994-3722

Article disponible en ligne à l'adresse :

https://www.cairn.info/revue-spirale-revue-de-recherches-eneducation-2017-1-page-27.htm

Distribution électronique Cairn.info pour Association pour la Recherche en Éducation.

(c) Association pour la Recherche en Éducation. Tous droits réservés pour tous pays.

La reproduction ou représentation de cet article, notamment par photocopie, n'est autorisée que dans les limites des conditions générales d'utilisation du site ou, le cas échéant, des conditions générales de la licence souscrite par votre établissement. Toute autre reproduction ou représentation, en tout ou partie, sous quelque forme et de quelque manière que ce soit, est interdite sauf accord préalable et écrit de l'éditeur, en dehors des cas prévus par la législation en vigueur en France. Il est précisé que son stockage dans une base de données est également interdit. 


\title{
ENTRE RUPTURES ÉDUCATIVES ET CLOISONNEMENTS PÉDAGOGIQUES
}

\author{
REPENSER LA LUTTE CONTRE LE DÉCROCHAGE SCOLAIRE \\ EN LYCÉE PROFESSIONNEL
}

\begin{abstract}
Résumé : L'article présente l'analyse des effets d'un dispositif de lutte contre le décrochage scolaire, le SAS, déployé au sein d'un lycée professionnel. Il s'agit d'un dispositif d'orientation décalée à l'attention d'élèves de CAP. Une enquête de terrain menée au cours d'une année scolaire a permis de recueillir des données quantitatives auprès de la direction de l'établissement et des données qualitatives d'observations en classe et d'entretiens avec les élèves et les enseignants. L'originalité de cette recherche tient dans le regard croisé sur le dispositif et les différents acteurs impliqués. Nos résultats révèlent l'importance du processus de décrochage de l'intérieur chez les élèves, imposant aux enseignants de réinventer leurs pratiques pédagogiques. La discussion porte sur le risque de décrochage professionnel des enseignants que peut engendrer une telle situation empreinte de nouveaux défis.
\end{abstract}

Mots-clés : Décrochage scolaire, persévérance scolaire, réclusion scolaire, dispositif, activité enseignante, lycée professionnel

\section{INTRODUCTION}

Depuis 40 ans, les réflexions menées en matière de politique éducative et les efforts qui les accompagnent au sein des établissements scolaires ont permis de réduire le nombre d'élèves quittant l'école sans qualification, passant de plus de 200000 dans les années 1970 à un peu moins de 140000 aujourd'hui selon la direction générale de l'enseignement scolaire (DGESCO, 2014). Néanmoins, ce recul du phénomène masque une réalité bien plus préoccupante. En effet, comme le souligne Glasmann (2011), le contexte socio-économique favorable de l'époque permettait à ces jeunes sans qualification d'accéder à l'emploi et de s'insérer socialement. Ce n'est plus le cas aujourd'hui. Pour Fortin et Picard (1999), la précarité sociale et professionnelle est fortement corrélée avec le décrochage scolaire des élèves. Or, tous les élèves ne sont pas également confrontés à ce risque : les élèves de la voie professionnelle constituent une population particulièrement fragile.

Les facteurs explicatifs du décrochage scolaire sont multiples (Blaya \& Hayden, 2003 ; Millet, 2004). Parmi les plus influents, les processus d'orientation scolaire ont un impact important. Les élèves scolarisés en lycée professionnel y arrivent rarement par choix et le plus souvent à la suite d'un parcours scolaire chaotique fait d'échecs à répétition. Cette orientation scolaire subie (Blaya, 2010) se conjugue à un manque de reconnaissance et de valorisation de leur formation aussi bien dans le monde du travail que dans la hiérarchie sociale. Pour Perron (1991: 18), il s'agit d'un «premier raté social homologué ». Mais le diplôme reste leur 
dernière chance avant une exclusion sociale annoncée, avant un avenir semé de « galères », de retours plus ou moins réussis dans des formations ultérieures. Paugam (2000 : 226) note que «si à la sortie de l'école les risques de déclassement sont importants pour les moins diplômés, notamment les titulaires d'un CAP ou d'un BEP, ils le sont encore davantage pour ceux qui sont privés de toute qualification et de tout diplôme ».

Dans un tel contexte, l'absentéisme se révèle être un phénomène croissant et important dans la voie professionnelle, pouvant traduire un désengagement des élèves de leur formation. Ce manque d'intérêt pour les études (Janosz, Archambault, Morizot \& Pagani, 2008) se révèle être un puissant révélateur du risque de décrochage scolaire des élèves. Pour Glasman (2003), il s'agit là d'une situation de « décrochage cognitif». Celui-ci est en fait la conséquence d'un long et lent processus au cours duquel s'est installée une forme d'infériorisation cognitive. Les difficultés d'apprentissage ont généré progressivement une phobie face aux tâches scolaires, phobie qui en retour a figé leurs capacités et les a amenés à « choisir » la posture du mauvais élève. Ces élèves construisent, petit à petit, autour d'eux une sorte de mur qui les empêche d'accéder aux connaissances et, plus largement, à la compréhension même de ce qu'on leur demande. Une telle expérience ne se vit pas sans souffrance et génère une perte de confiance qui s'inscrit «dans un rapport indéterminé à l'avenir » (Jellab, 2003 : 55). Progressivement, cet élève de la voie professionnelle intériorise une identité négative et intègre l'idée qu'il est «nul» et que « les choses de l'école» ne sont pas faites pour lui. Il est persuadé qu'il n'a aucune aptitude et n'entreprend par conséquent plus rien même si bien des tâches sont à sa portée. Nous sommes proches de ce que Seligman et Beagley appellent «l'impuissance apprise » (1975: 534) autrement dit de la réclusion scolaire (Gaillard, 2010). L'élève adopte alors des conduites d'évitement et de repli qui lui permettent de résister à la souffrance que provoque l'écart entre les attentes de l'école et l'idée qu'il se fait de ses propres possibilités.

Si la situation scolaire est source de souffrance et de dévalorisation, "l'inquiétude soulevée par les risques de marginalité, d'errance et de déviance » (Guigue, 1998 : 25) incite malgré tout l'élève reclus à rester au sein de l'école. La quitter reviendrait à prendre le risque de voir sa vie basculer. L'école est, pour l'élève, tout à la fois le lieu où se créent les réseaux d'amitié, celui qui permet de revendiquer une identité sociale mais également et paradoxalement celui où s'exprime une certaine forme de violence scolaire en ce qu'il lui renvoie l'image de ses échecs, de ses difficultés et de son incapacité à répondre aux exigences de l'école. Ces élèves suivent leur formation sans s'y impliquer, sans la rejeter non plus : ils sont à l'école mais ne s'acquittent pas de leurs tâches.

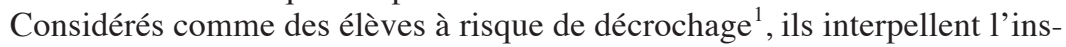
titution scolaire qui, d'une certaine manière, est responsable de leur devenir (Ministère de l'éducation nationale - MEN, 1999). Cette prescription institutionnelle se répercute sur le travail quotidien des professionnels de l'éducation, particulièrement les enseignants qui, dans leurs classes, sont amenés à gérer ces situations complexes pouvant conduire à une forme de décrochage professionnel. En effet, plusieurs études ont montré que ce dernier trouvait son origine, entre autre, dans

\footnotetext{
${ }^{1}$ Le décrochage correspond à l'état de fait, c'est-à-dire la situation avérée d'abandon d'étude sans avoir obtenu de diplôme et sans être en situation d'emploi. Le risque de décrochage correspond au processus, c'est-à-dire la situation d'un élève pour lequel plusieurs facteurs (absentéisme, résultats scolaire, comportement, manifestation d'intérêt pour les études, etc.) pourraient le conduire au décrochage.
} 
les rapports que les enseignants entretiennent avec l'institution et les élèves à risque de décrochage. L'augmentation forte, ces dernières années, de la prescription institutionnelle en matière de lutte contre le décrochage scolaire (Bruno, Méard \& Walter, 2013) engendre le sentiment chez les enseignants de ne plus se sentir en mesure d'y répondre du fait de la multiplicité des contraintes qui s'exercent sur leur métier (Lantheaume \& Hélou, 2008). En d'autres termes, l'institution scolaire est elle-même génératrice d'une démotivation des enseignants (GonthierMaurin, 2012). D'autres chercheurs considèrent ce risque de décrochage professionnel en lien étroit avec la complexité inhérente à la prise en charge des élèves en difficulté (Grossmann, 2011 ; Mukamurera \& Bouthiette, 2008).

Présentée «comme l'une des situations les plus problématiques des systèmes éducatifs actuels » (Moussay, 2014 : 146), la consubstantialité du risque de décrochage scolaire des élèves et professionnel des enseignants semble être un phénomène particulièrement marqué dans le contexte des lycées professionnels où se cumulent plusieurs des caractéristiques identifiées ci-dessus comme des facteurs de risque de ce double décrochage. Dans cet article, nous présentons les résultats d'une étude portant sur les effets de la mise en œuvre d'un plan de prévention du décrochage scolaire en lycée professionnel. Plus précisément, nous interrogeons ces effets à partir du regard croisé des ressentis des élèves et de l'activité des enseignants en situation de travail.

\section{LE CONTEXTE DE L'ÉTUDE}

L'établissement scolaire au sein duquel la recherche a été menée est situé en banlieue d'une ville moyenne française et subit pleinement le phénomène de décrochage scolaire et les problématiques qui l'accompagnent. Le constat était particulièrement alarmant pour les élèves scolarisés en Certificat d'aptitude professionnelle (désormais CAP). Pour l'année 2013-2014, on notait un taux d'absentéisme de 19,7\%, contre un taux moyen en lycée professionnel de 11,44\% et un taux de 35,7 \% de décrochage à l'issue du CAP, c'est-à-dire d'élèves ne parvenant pas à obtenir leur diplôme et se retrouvant à la rentrée suivante sans emploi ni formation alternative. Enfin, les formations de cet établissement semblent peu attractives avec des taux de pression ${ }^{2}$ se situant entre 0,48 et 1,75 (par exemple, Bac Pro Transport Logistique $[0,48]$ ou CAP Conducteur Routier $[0,80]$ ), alors qu'ils atteignent 5 et plus pour d'autres formations (par exemple la filière Esthétique cosmétique). En réponse à une telle situation, les personnels de direction, d'éducation et d'enseignement de l'établissement ont développé un plan d'action de lutte contre le décrochage scolaire.

Partant de l'hypothèse selon laquelle le défaut d'orientation, entre autres, impacte significativement le devenir scolaire des élèves de CAP, l'équipe pédagogique a mis en place à la rentrée 2014 le «SAS », un dispositif d'orientation décalée $e^{3}$ à l'attention des élèves de seconde CAP Métiers de 1'Automobile et de la Lo-

${ }^{2}$ Le taux de pression correspond au rapport entre le nombre de demandes d'entrée dans une formation et le nombre de places disponibles dans celle-ci. Il permet de cerner le degré d'attractivité d'une formation.

${ }^{3}$ Il s'agit d'un dispositif, sur une période de six semaines en tout début d'année scolaire, permettant aux élèves de $2^{\text {nde }} \mathrm{CAP}$ de travailler sur leur projet scolaire et professionnel et de découvrir les différentes formations. Ce travail est réalisé, à raison de quelques heures par semaine, sous l'accompagnement d'un enseignant référant. A l'issue de cette période, les élèves ont la possibilité de modifier leurs vœux d'affectation initiaux. A terme, l'ambition est de réduire le risque de décrochage scolaire en fa- 
gistique. Après la classe de $3^{\mathrm{e}}$ et dès leur arrivée au lycée, les élèves sont orientés vers un sas d'accueil leur permettant non seulement de choisir au mieux leur orientation mais aussi d'intégrer sereinement leur scolarité en lycée professionnel. Le «SAS » propose, sur une durée de 6 semaines en début d'année scolaire, un emploi du temps aménagé aux élèves de 2 CAP. Ils sont accompagnés par une équipe composée, pour l'occasion, de 8 professeurs référents, 3 conseillers principaux d'éducation (CPE) et de la direction. Dans le cadre de cette innovation, cette équipe se réunit chaque semaine pour penser, créer des outils, repérer les élèves en difficulté et réajuster la prise en charge.

Dès la première année, l'évolution des indicateurs chiffrés suggère des effets positifs à la mise en œuvre de ce dispositif. En effet, à l'issue de l'année 2014-2015, auprès de la population des élèves de CAP, on relève une baisse significative du taux d'absentéisme (15,6\% contre 19,7\% l'année précédente) ainsi que de celui de décrochage (13,6\% contre 35,7\% l'année précédente). Ces premières données semblent valider l'efficacité institutionnelle du dispositif (Ben Abid-Zarrouk, 2015) en ce sens que la mise en place de cette nouvelle procédure d'orientation a permis d'atteindre les objectifs fixés en termes de prévention du décrochage scolaire. Pour autant, elles ne disent rien de l'efficacité individuelle qui «suppose de mettre l'usager au centre du dispositif» (ibid. : 200). Dans ce contexte, nous avons été sollicités par l'équipe de direction de cet établissement pour accompagner le déploiement de ce dispositif et surtout en étudier les effets, notamment au plan qualitatif, auprès des élèves et des enseignants. En effet, aujourd'hui est acquise la conviction que la prévention du décrochage scolaire passe par une réflexion sur le phénomène en tant que processus s'inscrivant sur un empan temporel long (Blaya, 2010) et non sur les seules données statistiques.

\section{OPTIONS THÉORIQUES ET MÉTHODOLOGIQUES}

L'estimation de l'efficacité telle que mentionnée ci-dessus (réduction du taux de décrochage) tend à attribuer aux effets du dispositif l'évolution du taux de décrochage. Elle ne permet pas d'apprécier la part effectivement liée au dispositif de celle inhérente à des changements chez l'individu (par exemple un élève initialement désengagé pourra se remobiliser pour sa formation pour des motifs totalement étrangers au dispositif, par exemple des pressions exercées par son environnement familial). Or, la complexité et la multidimensionalité du phénomène du décrochage scolaire imposent une certaine prudence à l'égard de cette affirmation. Aussi avons-nous proposé une étude qualitative à partir d'un regard croisé portant sur une analyse de l'activité des enseignants en situation de classe et des trajectoires scolaires et personnelles des élèves. Il nous importait de pouvoir appréhender le point de vue des élèves sur leur situation scolaire en tenant compte de leur parcours et de l'historicité de leur relation à l'école. Ce choix incombe essentiellement au fait que le décrochage scolaire est un processus qui s'inscrit dans un empan temporel long. En revanche, parce que les enseignants «voient passer les élèves devant eux» (propos d'un élève de CAP), nous avons privilégié une entrée centrée sur l'activité enseignante dans l'ici et maintenant en référence à un cadre théorique permettant néanmoins de saisir la complexité de cette activité, et notamment dans l'articulation entre les éléments d'expérience et ceux plus circonstanciés.

vorisant l'affectation dans des filières choisies par les élèves. 
L'analyse de l'activité des enseignants en situation de classe est menée en référence aux postulats d'une théorie historico-culturaliste de l'activité (Engeström, 1987 ; Leontiev, 1976) et de la clinique de l'activité (Clot, 2008). Cette approche repose sur le postulat que la lutte contre le décrochage scolaire passe d'abord par une intervention de l'enseignant judicieuse, ajustée et ajustable, permettant aux élèves de renouer avec les apprentissages et que pour être comprise cette activité doit être étudiée au plus près des situations ordinaires. Les données recueilles pour cette étude consistaient en des données d'observation en classe et des données de verbalisation sur la base d'entretiens semi-directifs individuels (entretiens compréhensifs, auto-confrontations et instructions au sosie ${ }^{4}$ ) et collectifs (entretiens de groupe et auto-confrontations croisées) réalisés avec trois enseignants (deux enseignants de lettres/histoire-géographie (enseignante N. et enseignant P.) et une enseignante de Prévention Sécurité Environnement (Enseignante S) constituant le collectif de travail. L'analyse de ces entretiens a permis l'identification des ressorts de l'activité des enseignants en situation de travail pour appréhender ce qu'ils mettent en œuvre pour prévenir et lutter contre le décrochage scolaire et, à terme, soutenir le développement de leur pouvoir d'agir (Flavier, Moussay \& Méard, 2015).

Enfin, les trajectoires scolaires et personnelles des élèves ont fait l'objet d'un deuxième volet de notre regard croisé sur l'impact du «SAS » et du plan d'action de lutte contre le décrochage scolaire ainsi que sur le processus de persévérance scolaire auprès de ces élèves à risque. À cette fin, des entretiens semidirectifs ont été conduits afin, en tenant compte du temps d'administration possible, c'est-à-dire une séquence dont la durée n'excède pas 50 minutes, de recueillir les représentations, les attentes des élèves, d'identifier et de comprendre les mécanismes d'entrée dans le décrochage scolaire. Autant que possible, avons-nous laissé l'interviewé parler ouvertement, dans les mots qu'il souhaitait et dans l'ordre qui lui convenait. Quand l'entretien s'écartait des thèmes de la recherche, nous reposions des questions auxquelles l'interviewé ne venait pas par lui-même. Le corpus des entretiens (retranscrit in extenso) a fait l'objet d'une analyse par catégorisation à partir des mots-clés du discours des élèves, afin de repérer, dans leurs propos, les éléments permettant de dresser leur profil à partir de leur parcours scolaire, de leurs ressentis sur celui-ci et sur leur orientation actuelle.

\section{RÉSULTATS}

Si les repères statistiques semblent indiquer un effet positif sur la persévérance scolaire des élèves, l'analyse des données qualitatives révèle toute la complexité de la prise en charge du risque de décrochage scolaire. En effet, s'ils restent en plus grand nombres scolarisés et manifestent un certain désir d'apprendre, les élèves renvoient également l'image de jeunes peu investis et doutant d'eux-

\footnotetext{
${ }^{4}$ Mise au point par l'ergonome italien, Oddone, l'instruction au sosie est une technique d'entretien permettant d'extraire les savoirs professionnels à partir d'une description précise de l'activité par son auteur. Elle a pour point de départ la consigne suivante : "Suppose que je sois ton sosie et que demain je me trouve en situation de te remplacer dans ton travail. Je te questionne sur la manière dont je dois agir, en insistant sur les détails ». L'interviewer (ici le chercheur) s'attache ensuite à soutenir l'acteur (ici l'enseignant) dans la délivrance des instructions les plus précises possible quant à la réalisation de son travail. L'analyse de l'entretien permet d'accéder à une compréhension de l'activité, en adoptant le point de vue de l'acteur, à un grain extrêmement fin. Pour un exemple de cette technique, voir Clot et Soubiran (1998).
} 
mêmes. En regard, les enseignants vivent des situations professionnelles marquées par des conflits de logiques entre d'une part l'obligation de répondre aux exigences institutionnelles et d'autre part l'ambition de soutenir les élèves dans leur parcours de formation. Ces tensions dans le travail enseignant sont présentées en deux points.

\section{Quand faire réussir l'élève passe par des accommodements évaluatifs}

L'enfermement des élèves dans une forme de passivité apparente, pouvant être perçue comme de «l'anorexie scolaire » ou de «l'inappétence » est en réalité le reflet d'une stratégie de fuite face à un nouvel échec qu'ils redoutent. Mais lorsqu'ils ont la parole, c'est bien un droit à participer et à réussir que ces élèves revendiquent :

« Aller à l'école... c'est pour avoir un diplôme... c'est pour être bien... pour apprendre et devenir quelqu'un... apprendre pour savoir plus de choses... c'est mieux d'être chez les grands »

(Extrait entretien avec Mathieu $\left.{ }^{5}\right)$

« Après avoir un avenir... un travail euh... plus tard... d'avoir mon CAP... si il n'y avait pas le CAP je viendrais quand même... parce qu'il vaut mieux apprendre des trucs... c'est toujours bien d'apprendre »

(Extrait entretien avec Mara)

«Pour apprendre... à l'école on peut apprendre c'est tout. La plupart ils ne savent pas ce qu'ils font là. Moi j'aurais préféré faire un apprentissage »

(Extrait entretien avec Jonathan)

Cette ambition n'est pas toujours exprimée aussi clairement. Pour d'autres élèves, le malentendu culturel sur la signification de ce qui est à faire à l'école perdure. Quand ils pensent avoir fourni les efforts pour réussir sans pour autant que cette réussite se réalise, le sentiment de l'inutilité s'installe :

«... à rien il faut venir c'est tout... de toute façon c'est quand même mieux que de rester à la "ker" [la maison] mais si je n'étais pas obligé je ne viendrais pas tous les jours...»

(Extrait entretien avec Damien)

«... aller à l'école ?... je ne sais pas j'y vais parce que... on n'a pas le choix... on est obligé... et puis si on n'y va pas on traîne dans la rue... on peut faire des bêtises... »

(Extrait entretien avec Valentin)

En réalité, ce sont surtout la norme sociale et le désir d'apprendre qui placent ces élèves sur le chemin de l'école. Pour la plupart d'entre eux, âgés d'au moins 16 ans, la scolarisation n'est plus obligatoire. Ces témoignages traduisent l'ambiguïté de la posture des élèves, pris en tenaille entre le désir de réussir d'une part et le sentiment de ne pas en être capable d'autre part. Dès lors, en réponse aux stratégies d'évitement des élèves, les enseignants sont amenés à « négocier » les règles d'exercice de leur métier afin de pallier la précarité de cette relation professeur-élève. Cette situation s'observe dans les arbitrages que les enseignants réalisent autour de la passation du contrôle en cours de formation ${ }^{6}$ (CCF).

\footnotetext{
${ }^{5}$ Afin de préserver l'anonymat des personnes (élèves et enseignants), des prénoms de substitution sont utilisés.

${ }^{6}$ Le contrôle en cours de formation permet une adaptation de fait de la formation et de sa validation aux particularités locales. Il prend la forme de deux ou trois situations d'évaluation organisées en établissement. Sa conception et sa mise en œuvre sont de la responsabilité des formateurs de l'élève. Il intervient à des moments clés de la formation, en fonction des acquisitions des élèves. Il a pour objectif
} 
Le pilotage de l'enseignement par la certification (Eckert \& Veneau, 2000) incite les enseignants à penser leurs cours pour répondre à l'évaluation qu'ils ont eux-mêmes construite. Plus précisément, ils cherchent à « orienter » le travail des élèves lors des cours qui précèdent la date du CCF. C'est le cas de l'enseignante S., en Prévention Sécurité Environnement, qui

« insiste sur les explications à propos de l'IMC [Indicateur de Masse Corporelle] parce qu'ils ont eu des difficultés pendant le cours et je sais que je l'ai mis dans le CCF » [elle argumente] «En plus ce cours, je ne l'ai pas fait avec eux, ils l'ont vu pendant le forum santé, alors je ne veux pas qu'ils aient l'impression d'être pris au piège mais si je dis rien, si je rappelle pas, si je donne pas des exemples, ils ne vont pas y arriver, alors je les aide un peu, je heu... j'oriente un peu leurs révisions sans vraiment leur dire, en leur disant que c'est important, en insistant »

(Extrait entretien d'auto-confrontation avec l'enseignante S.)

Son activité relève d'un compromis opératoire, à l'interface de deux prescriptions qu'elle perçoit au départ comme antinomiques. Il est attendu que les élèves puissent présenter le CCF au moment de leur choix, dès qu'ils se sentent en mesure de le réussir (prescription 1). Néanmoins cette situation implique de composer autant de sujets qu'il y a d'élèves (prescription 2), ce qui, se révèle intenable et est vécu par l'enseignant comme un conflit. Afin de dépasser ce dernier et favoriser les chances de réussite à l'examen (motif d'agir 1) dont la date, commune à tous les élèves est fixée par elle, l'enseignante leur suggère des pistes de révision (but) en revenant avec insistance sur certaines notions du programme (opération), en l'occurrence l'IMC, selon elle mal maîtrisée par les élèves. Elle agit ainsi, notamment, pour ne pas hypothéquer la confiance qu'elle a nouée avec les élèves (motif d'agir 2). En effet, les interroger sur une notion qu'elle n'a pas abordée directement avec eux, mais qu'ils ont découverte dans le cadre du forum santé pourrait être interprété par les élèves comme une «trahison» pour reprendre les termes de l'enseignante. Ainsi, nous observons ici un premier arbitrage qui consiste à s'accommoder avec la règle du secret sur le contenu de l'évaluation, sans pour autant le révéler de manière explicite.

Pour l'enseignante N., enseignante de lettres/histoire-géographie, le fort taux d'absentéisme lors des épreuves du CCF alors même que les élèves en ont choisi la date est un indicateur de leurs stratégies de fuite. Cet évitement est le reflet d'une crainte de l'échec qu'elle contrecarre en évaluant les élèves selon des dispositions aux frontières de celles définies par l'institution :

« il m'arrive de les évaluer sans vraiment qu'ils le sachent, je leur dis que l'on fait un entraînement au CCF, juste comme ça, pour voir s'ils sont capables et parfois, si c'est réussi je le garde et je leur dis "tu vois que tu es capable, tu as réussi" et cela leur redonne confiance, cela leur montre qu'ils peuvent réussir, qu'ils sont pas toujours en échec, c'est important pour eux, parce que sinon, ils risquent de ne plus venir du tout, et ça, il faut l'éviter car ils se retrouvent sans diplôme, on peut pas les rattraper tout le temps »

(Extrait entretien d'auto-confrontation avec l'enseignante N.).

À nouveau les propos de cette enseignante mettent en exergue les tensions qui l'animent dans l'exercice de son métier. Les accommodements évaluatifs, à savoir retenir les prestations des épreuves d'entraînement pour valider le CCF sans en avoir fait préalablement part aux élèves, se révèle être un compromis judicieux

de rendre plus proches l'évaluation et la formation et de permettre de répartir l'évaluation dans la durée. 
lui permettant d'atténuer la pression de l'évaluation tout en respectant les conditions de l'examen (moment de passation individualisé, format de l'épreuve). Un tel accommodement, qui pourrait passer pour une «trahison » aux yeux de l'élève, constitue cependant une circonstance propice à la réussite de ce dernier et surtout offrant à l'enseignante la possibilité de mettre l'élève face à ses réussites.

\section{Des acquisitions méthodologiques pour redonner confiance}

Le dispositif «SAS » permet aux élèves de s'orienter vers une formation de leur choix, du moins parmi celles proposées par cet établissement. Cette possibilité, reposant sur le droit à l'erreur (l'orientation finale peut être différente de celle pressentie lors de la rentrée), vise à redonner une certaine confiance en l'institution scolaire pour ces élèves au parcours souvent chaotique. Leurs propos montrent également qu'ils n'ont pas perdu tout espoir. Ils expriment un besoin d'aide :

Chercheur (Ch) : As-tu appris beaucoup de choses depuis que tu es arrivé ici ?

Élève (E) : Oui... j'ai appris beaucoup trop de respect... j'ai appris l'atelier... j'ai appris que les profs étaient en soutien...

Ch : Quel est le métier que tu aimerais exercer plus tard ?

(Extrait entretien avec Mathieu)

E :...je pense pas être prof... parce que c'est trop dur... je pourrais le faire euh... dans les ateliers... pour être prof d'atelier...

$\mathrm{Ch}: \mathrm{Qu}$ 'est ce qui t'aiderait à mieux apprendre?

$\mathrm{E}$ : Qu'on m'aide... qu'on me soutienne plus

$\mathrm{Ch}$ : Tu n'es pas assez soutenue? profs seuls...

$\mathrm{E}:$ Si mais un peu plus çà serait mieux... c'est déjà bien on peut parler avec les

(Extrait entretien avec Mara)

E :...parce que nos profs d'atelier ils sont toujours à l'écoute... ils sont toujours là... ils ont... ils ont pas que nous mais... quand ils sont toujours... mais quand on ne va pas bien ils savent... ils savent ce qu'on a...

(Extrait entretien avec Valentin)

Les enseignants ne s'y trompent pas, ces élèves ont besoin d'être soutenus et accompagnés dans leur scolarité dès lors que la réduction du risque de décrochage scolaire s'affirme comme l'un des objectifs prioritaires du projet d'établissement. Néanmoins, cette situation n'est pas sans effet sur l'activité des enseignants. Ils éprouvent le sentiment de «tenir les élèves à bout de bras » et se sentent contraints de «perdre du temps » (extrait entretien d'auto-confrontation avec l'enseignante N.) en début d'année pour réapprendre aux élèves à organiser la tenue de leurs cours et prises de notes. Or ce déficit dans l'appropriation de ces acquisitions méthodologiques opère comme un puissant levier dans le risque de décrochage car, très vite, les élèves se retrouvent perdus et dans l'incapacité de raccrocher aux cours. Cela génère un certain nombre de désillusions. Ainsi Valentin voyant ses espérances scolaires déjouées, regrette de ne pas avoir plus travaillé :

«...pour mon avenir... quand on me disait il faut que tu travailles pour ton avenir... je ne savais pas... du coup je suis venu en mécanique en attendant de faire de la peinture » ou Nael qui «voulai[t] faire un apprentissage... un apprentissage... et [n'a] pas trouvé alors [il a] été obligé de venir ici ».

Chaque enseignant développe ses propres stratégies d'aide et d'accompagnement afin de remobiliser les élèves sur le travail scolaire. Au-delà des enjeux psychosociaux, voire quelques fois des savoirs disciplinaires, ce sont les acquisitions méthodologiques qui deviennent prédominantes : apprendre à travailler, à 
s'organiser, à tenir ses affaires de classe sont des prérequis à la réussite scolaire que ces élèves peinent à maîtriser.

Lors d'un cours de géographie avec une classe de seconde CAP, l'enseignante $\mathrm{N}$. invite les élèves à colorier un fond de carte de Chine selon un code couleur défini afin de représenter les inégalités économiques. Bien qu'importante, la notion géographique sert ici de support. L'enseignante ambitionne que les élèves «puissent être en mesure de schématiser une idée, de se la représenter dans leur tête » parce que «la difficulté pour eux réside dans le soin, se repérer, essayer d'organiser eux-mêmes les couleurs par exemple, passer du plus foncé au plus clair par exemple et pour eux c'était pas évident, c'était pas si simple et ça pourra leur servir à d'autres moments ». Dans ce court extrait, l'enseignante révèle des motifs d'agir qui ne sont pas directement attachés aux acquisitions géographiques, mais bien à des compétences que les élèves pourront ré exploiter à court terme dans le cadre de leur scolarité ou des enseignements professionnels.

Cette préoccupation est partagée par ce professeur de français, l'enseignant P., qui, à la suite du visionnage d'un film demande aux élèves d'associer des images extraites du film aux principales idées du scénario ici résumé en une quinzaine de lignes. Pendant plus de 20 minutes, il circule dans les rangs, interrogeant les élèves, tolérant des discussions entre eux, avant de proposer un temps collectif de «correction». Une fois encore, l'association image-idée n'est qu'un prétexte. Derrière la forme presque enfantine de ce travail se cachent des attentes liées à un «travail de l'argumentation parce qu'ils ont l'impression de connaître le film mais, en réalité, ils ont du mal à dire pourquoi ils ont choisi telle image, il faut qu'ils s'appuient sur des détails, qu'ils m'expliquent ». À nouveau, le support sert de prétexte : la capacité de l'élève à résumer le film doit permettre de travailler l'argumentation. Par ailleurs, dans la classe, tout est fait pour que les élèves se concentrent sur la tâche demandée : s'ils disposent tous d'un document présentant le résumé et les images, il est également projeté au mur; si l'enseignant accorde un temps long à ce travail c'est pour permettre à «chaque élève de s'y engager individuellement et parce qu'ils ont besoin de temps pour se mettre au travail, quand tu leur donnes quelque chose à faire, ils papillonnent, ils traînent, tu perds toujours du temps, mais au final, ce qui compte c'est qu'ils réfléchissent chacun, même si c'est juste quelques minutes au cours de la leçon ». Les motifs d'agir de l'enseignant sont plus profonds que ne le laisse penser l'observation de la situation. Si le temps long et l'engagement de l'enseignant dans des interactions interindividuelles offrent, inéluctablement, aux élèves des opportunités de se divertir, ils constituent également une condition sine qua non à la satisfaction des motifs d'agir de l'enseignant qui se situent dans des acquisitions transversales (ici l'argumentation) jugées essentielles au devenir de l'élève en qualité de futur adulte et travailleur.

\section{DISCUSSION}

Les résultats de notre étude ouvrent deux points de discussion. Ils permettent de réinterroger les typologies des élèves décrocheurs, et complémentairement, d'envisager l'impact de la prise en charge du décrochage scolaire sur le décrochage professionnel des enseignants.

De nombreux travaux, principalement d'inspiration sociologique, ont proposé différentes typologies permettant de repérer les élèves à risque de décrochage (Bernard, 2011 ; Bonnery, 2007 ; Janosz, 2000). Si ces typologies reposent notamment sur l'étude des multiples facteurs qui tendent à entraîner un décrochage des 
élèves (facteurs sociaux, personnels, économiques, etc.), elles mettent également en exergue une certaine forme de responsabilité de l'institution scolaire. Le décrochage n'apparaît pas seulement être le reflet au sein de l'école de problématiques extrascolaires ; il est également induit par des phénomènes propres à l'école comme le confirment nos résultats. Les témoignages d'élèves que nous avons analysés montrent qu'ils éprouvent le sentiment de ne pas être les artisans de leur orientation scolaire et d'être ainsi dirigés vers des filières qui ne correspondent pas forcément à leurs ambitions ou à leurs désirs. En cela, les élèves que nous avons rencontrés tendent à «décrocher sur place » et s'apparentent aux «exclus de l'intérieur» décrits par Bourdieu et Champagne (1992: 73). Pour ces auteurs, l'école « exclut désormais de manière continue, à tous les niveaux du cursus [...] et elle garde en son sein ceux qu'elle exclut, se contentant de les reléguer dans des filières plus ou moins dévalorisées $»$. Cette tendance semble particulièrement forte dans le contexte de l'enseignement professionnel de notre étude, établissement au sein duquel les taux de pression relativement faibles révèlent un manque d'intérêt, a priori, des élèves pour les formations dispensées. Or, l'orientation, lorsqu'elle est subie par l'élève, opère comme un facteur augmentant le risque de décrochage de ce dernier (Blaya, 2010) et dont les effets se font sentir, à plus long terme, en hypothéquant son insertion professionnelle (Boudesseul, 2010 ; Brinbaum \& Guégnard, 2012).

Nos résultats tendent à montrer également que ce phénomène de décrochage de l'intérieur n'échappe pas totalement aux enseignants et qu'il se révèle sans doute moins discret qu'il n'y paraît. En effet, nous avons pu identifier les efforts déployés par les enseignants pour favoriser la réussite des élèves, notamment dans le cadre du CCF ou pour créer des circonstances propices à un engagement minimum de chacun, notamment en leur octroyant du temps et en multipliant les supports. Les enseignants revendiquent prendre en considération les difficultés des élèves et agir en faveur d'une revalorisation de leur travail scolaire. Mais nos résultats montrent également l'impact sur le travail enseignant. Un tel contexte est à l'origine de situations de travail contrariées (Clot, 2010) en ce sens que l'activité des enseignants se trouve dévitalisée d'une partie de son sens. Le sentiment qu'ils éprouvent, d'incapacité à faire réussir tous les élèves en dépit de leurs efforts, affecte la pérennité de leurs motifs d'agir initiaux en lien avec les missions éducatives qui leur sont confiées. Malmenés par l'étendue des contraintes et prescriptions qui s'imposent à eux, les enseignants éprouvent des difficultés à concilier les attentes de l'institution scolaire et leurs ambitions, en termes d'accompagnement et de soutien aux élèves, que leurs convictions leur suggèrent. À cette fin, le métier traditionnel ne suffit plus et les enseignants doivent puiser dans le collectif les ressources à un développement de leur pouvoir d'agir (Flavier \& Méard, sous presse), c'est-à-dire réinventer leurs pratiques professionnelles ou supporter les accommodements locaux que nous avons évoqués et qui ne sont pas sans rappeler les caractéristiques des arrangements évaluatifs (Merle, 1996). À la problématique du risque de décrochage scolaire des élèves s'ajoute alors celle du risque de décrochage professionnel des enseignants dès lors qu'ils ne parviennent pas à réaliser leur métier dans les « règles de l'art » (Roger, 2007) ni ne trouvent les ressources à un développement professionnel.

En conclusion, fort de ce regard croisé, il apparaît que les enseignants se heurtent trop souvent à des résistances de la part des élèves qui ne parviennent pas à saisir les enjeux de ces formes de travail, de ces exigences, qu'ils perçoivent surtout comme des contraintes scolaires. Nael «avai $[\mathrm{t}]$ déjà tout appris au collège [et n'a] pas l'impression d'apprendre quelque chose ». Pierre est plus sévère, il «ai- 
merai $[\mathrm{t}]$ apprendre des choses utiles quoi... des... des choses qui servent dans la vie... je ne sais pas moi... mais bon... pour avoir un bon métier ». Un tel constat révèle une forme d'incompréhension mutuelle entre enseignants et élèves, engendrant le risque d'un double décrochage (Karsenti \& Collin, 2009) : celui, scolaire, des élèves du fait de la répétition des échecs et de l'ennui ; celui, professionnel, des enseignants inhérent au sentiment d'abord d'épuisement puis d'impuissance qu'ils éprouvent.

Éric FLAVIER

LISEC (EA 2310)

ESPE de l'académie de Strasbourg

Université de Strasbourg

Joël GAILLARD

LISEC (EA 2310)

Université de Nancy

Sandoss BEN ABID-ZARROUK

LISEC (EA 2310)

Université de Haute Alsace

\begin{abstract}
The article presents the analysis of the impact of a device designed to counter early school dropout, the SAS, deployed in a professional high school. This is an offset orientation device for students in professional training. À field survey conducted during a school year has collected quantitative data from the school management and qualitative data from classroom observations and interviews with students and teachers. The originality of this research lies in the mixed method over the device and the different actors involved. Our results reveal the importance of the internal processing of school dropping out, requiring teachers to renew their teaching practices. The discussion concerns the risk of professional dropout of the teachers that such a situation marked with new challenges can create.
\end{abstract}

Keywords : school dropout, school retention, school reclusion, specific measures, teacher activity, vocational school.

\title{
Bibliographie
}

Ben Abid-Zarrouk S. (2015) Estimer l'efficacité en éducation. Paris : L'Harmat$\tan$.

Bernard P.Y. (2011) «Le décrochage des élèves du second degré : diversité des parcours, pluralité des expériences scolaires »-Les Sciences de l'Éducation - Pour l'Ère Nouvelle 45 (75-97).

Blaya C. \& Hayden C. (2003) Construction sociale du refus de l'école : processus de non-scolarisation, de déscolarisation et de décrochage scolaire en France et en Angleterre. Rapport de recherche pour la Direction de la Programmation et du Développement (2000-012).

Blaya C. (2010) Décrochages scolaires. L'école en difficultés. Bruxelles: de Boeck.

Bonnery S. (2007) Comprendre l'échec scolaire. Élèves en difficultés et dispositifs scolaires. Paris : La Dispute.

Boudesseul G. (2010) «La segmentation par l'orientation? Comment l'orientation préfigure les inégalités d'emploi »-Formation Emploi. Revue Française de Sciences Sociales 109 (53-70). 
Bourdieu P. \& Champagne P. (1992) «Les exclus de l'intérieur» - Actes de la Recherche en Sciences Sociales 91-92 (71-75).

Brinbaum Y., \& Guégnard C. (2012) «Parcours de formation et d'insertion des jeunes issus de l'immigration au prisme de l'orientation »-Formation Emploi 2 (61-82).

Bruno F., Méard J. \& Walter E. (2013) «Les dispositifs français de lutte contre le décrochage scolaire en collège : ce qui est prescrit et ce qui est mis en œuvre » - L'orientation Scolaire et Professionnelle 42 (439-459).

Clot Y. (2008) Travail et pouvoir d'agir. Paris : PUF.

Clot Y. (2010) Le travail à cour. Pour en finir avec les risques psychosociaux. Paris : La Découverte.

Clot Y. \& Soubiran M. (1998) «"Prendre la classe" : une question de style ? »Société Française 12/13 (78-88).

DGESCO (2014) Évaluation partenariale de la politique de lutte contre le décrochage scolaire. Rapport de diagnostic, mars 2014.

Eckert H. \& Veneau P. (2000) «Le rapprochement de l'école et de l'entreprise dans l'enseignement technique : sur les limites d'une rationalisation volontariste »-RFP 131 (33-41).

Engeström Y. (1987) Learning by expanding : an activity-theoretical approach to developmental research. Helsinki : Orienta-Konsultit.

Flavier É. \& Méard J. (sous presse) «L'approche du décrochage scolaire selon les théories culturalistes de l'activité. Bénéfices et perspectives »@ctivité.

Flavier E., Moussay S. \& Méard, J. (2015) «Les initiatives individuelles au cœur des dispositifs collectifs locaux de prévention du décrochage scolaire » - Les Dossiers des Sciences de l'Éducation 33 (175-192).

Fortin L. \& Picard Y. (1999) «Les élèves à risque de décrochage scolaire : facteurs discriminants entre décrocheurs et persévérants »-Revue des Sciences de l'Éducation 25 (359-374).

Gaillard J. (2010) Comprendre la réclusion scolaire. Paris : L'Harmattan.

Glasman D. (2003) «Quelques acquis d'un programme de recherches sur la déscolarisation »-Ville École Intégration 132 (8-18).

Glasman D. (2011) Le décrochage scolaire. Paris : PUF.

Gonthier-Maurin B. (2012) Rapport d'information fait au nom de la commission de la culture, de l'éducation et de la communication par la mission d'information sur le métier d'enseignant. Rapport Sénat, 601, session ordinaire 2011-2012.

Grossmann S. (2011) «Des enseignants qui res(is)tent : dynamiques identitaires et investissement du champ de l'enseignement professionnel au Québec »-Recherches en Éducation 11 (67-82).

Guigue M. (1998) «Le décrochage scolaire»-in : M. C. Bloch \& B. Gerde (éds.) Les lycéens décrocheurs. De l'impasse aux chemins de traverse (2538). Lyon : Chronique sociale.

Janosz M. (2000) «L'abandon scolaire chez les adolescents : perspective NordAméricaine »-VEI enjeux, 122 (105-127).

Janosz M., Archambault I., Morizot J. \& Pagani L. S. (2008) «School engagement trajectories and their differential predictive relations to dropout »- Journal of Social Issues 64 (21-40).

Jellab A. (2003) «Entre socialisation et apprentissages : les élèves du lycée professionnel à l'épreuve des savoirs » - RFP 142 (55-67). 
Karsenti T. \& Collin S. (2009) «L'autre décrochage scolaire» - Formation et Profession 16 (2- 6).

Lantheaume F. \& Hélou C. (2008) La souffrance des enseignants. Une sociologie pragmatique du travail enseignant. Paris : PUF.

Leontiev A. (1976) Le développement du psychisme. Paris : Éditions sociales.

MEN (1999) Circulaire n 99-071, Programme « Nouvelles Chances».

Merle P. (1996) L'évaluation des élèves. Enquête sur le jugement professoral. Paris : PUF.

Millet M. (2004) « Ruptures familiales et "ruptures scolaires" de collégiens de milieux populaires »-Actes du colloque : Le devenir des enfants de familles défavorisées en France. Paris.

Moussay S. (2014) «Le double décrochage au cœur des échanges sur l'activité en classe »- in : É. Flavier et S. Moussay (dir.) Répondre au décrochage scolaire. Expériences de terrain (145-154). Louvain-la-Neuve : De Boeck.

Mukamurera J. \& Bouthiette M. (2008) «Rester dans l'enseignement ou quitter ? Portrait de la situation et motivation des enseignants ». Communication au séminaire «Pourquoi et comment soutenir l'insertion professionnelle de nouveaux enseignants au Québec? Résultats de recherche et pistes d'action » Québec : Université de Sherbrooke.

Paugam S. (2000) La disqualification sociale. Paris : PUF.

Perron R. (1991) Les représentations de soi. Toulouse : Privat, Pratiques sociales.

Roger D. (2007) Refaire son métier. Essais de clinique de l'activité. Toulouse: Érès.

Seligman M. \& Beagley G. (1975) Helplessness : on depression, development and death. San Fransisco : Freeman. 